\title{
Breakdown of Fermi liquid behavior near the hot spots in a two-dimensional model: A two-loop renormalization group analysis
}

\author{
Vanuildo S. de Carvalho ${ }^{a}$, Hermann Freire ${ }^{a, b, *}$ \\ a Instituto de Física, Universidade Federal de Goiás, 74.001-970 Goiânia, GO, Brazil \\ ${ }^{\mathrm{b}}$ Department of Physics, Massachusetts Institute of Technology, Cambridge, MA 02139, USA
}

Received 29 April 2013; received in revised form 11 July 2013; accepted 17 July 2013

Available online 24 July 2013

\begin{abstract}
Motivated by a recent experimental observation of a nodal liquid on both single crystals and thin films of $\mathrm{Bi}_{2} \mathrm{Sr}_{2} \mathrm{CaCu}_{2} \mathrm{O}_{8+\delta}$ by Chatterjee et al. [Nature Phys. 6 (2010) 99], we perform a field-theoretical renormalization group (RG) analysis of a two-dimensional model such that only eight points located near the "hot spots" on the Fermi surface are retained, which are directly connected by spin density wave ordering wavevector. We derive RG equations up to two-loop order describing the flow of renormalized couplings, quasiparticle weight, several order-parameter response functions, and uniform spin and charge susceptibilities of the model. We find that while the order-parameter susceptibilities investigated here become non-divergent at two loops, the quasiparticle weight vanishes in the low-energy limit, indicating a breakdown of Fermi liquid behavior at this RG level. Moreover, both uniform spin and charge susceptibilities become suppressed in the scaling limit which indicate gap openings in both spin and charge excitation spectra of the model.
\end{abstract}

(c) 2013 Elsevier B.V. All rights reserved.

Keywords: Field-theoretical renormalization group; Strongly correlated electronic systems; Non-Fermi liquids;

Two-dimensional fermionic models

\footnotetext{
* Corresponding author at: Department of Physics, Massachusetts Institute of Technology, Cambridge, MA 02139, USA.

E-mail address: hfreire@mit.edu (H. Freire).
} 


\section{Introduction}

The nature of the pseudogap state which manifests itself in underdoped cuprates continues to generate both interest and controversy in the field of high- $T_{c}$ superconductivity. This is because the proper understanding of this phase turns out to be crucial for comprehending the underlying mechanism of superconductivity displayed by these materials [1,2]. In recent years, angle-resolved photoemission spectroscopy (ARPES) shed new light on this problem. Experiments [3] performed at very low temperatures by Chatterjee et al. on both single crystals and thin films of $\mathrm{Bi}_{2} \mathrm{Sr}_{2} \mathrm{CaCu}_{2} \mathrm{O}_{8+\delta}$ gave important evidence pointing to the existence of an electronic state - located in between the antiferromagnetic (AF) and $d$-wave superconducting (SC) phases - whose excitation spectrum becomes zero only at the so-called nodal points (i.e. in the direction along the line connecting $\Gamma=(0,0)$ and $M=(\pi, \pi)$-points in momentum space). This state has thus been called a nodal liquid [4-6]. This electronic state is insulating but with a $d$-wave gap structure and exhibits non-Fermi liquid behavior around the so-called "hot spots" (i.e. points in momentum space where the AF zone boundary intersects the underlying Fermi surface of the system).

A minimal electronic model which potentially contains the rich phenomenology of the phase diagram displayed by the cuprates is the two-dimensional (2D) Hubbard model on a square lattice. This conjecture, originally put forward by Anderson [2] in the mid 80s, sparked among other things a profound reexamination of the foundations of Landau Fermi-liquid theory and, particularly, the precise conditions in which this standard theory breaks down in some 2D strongly correlated models. Indeed, soon after the experimental discovery of the cuprate superconductors, Anderson proposed that the ground state of the 2D Hubbard model slightly away from halffilling is given by an insulating spin liquid (ISL) which has no broken symmetries down to zero temperature due to strong quantum fluctuations and also exhibits no well-defined quasiparticle excitations at low energies $[7,8]$. This question, however, remains unresolved to this date. Despite that, these ISL states are very interesting from a theoretical viewpoint due to the fact that, once they are lightly doped with holes, they indeed give rise to a singlet $d$-wave superconducting phase at mean-field level [9] in agreement with experimental observation.

On the other hand, for a long time, there has not been a rigorous proof of the stability of this ISL state in realistic microscopic models. Part of this imbroglio owes to the fact that, at the present time, there is no general analytical or numerical technique that allows one to solve in a exact (or nearly exact) way such strongly correlated models in 2D. In the last years, important advances have been made with improvements in both analytical methods (see, e.g., Refs. [10-15]) and also numerical techniques [16-18] which, supplemented by groundbreaking experimental results [19-21], provided strong evidence for the existence of these new quantum states in some realistic physical situations.

From a weak-to-moderate coupling perspective, renormalization group (RG) methods remain one of the most powerful tools to attack these problems in view of its unbiased nature [22-24]. They have been applied to many electronic models ranging from simpler 2D models [25-31] which focus on the dominant role of scattering processes involving Fermi surface regions of the model to the fully 2D Hubbard model defined on different lattice types. Regarding the 2D Hubbard model on a square lattice for weak-to-moderate couplings, for instance, some of these RG works successfully reproduced both at one-loop [32-34] and two-loop orders [35,36] an AF phase in the model near half-filling, the onset of a $d$-wave singlet SC phase away from half-filling, and an additional electronic phase reminiscent of the pseudogap state $[35,37,38]$ which interpolates between these two phases. This agrees qualitatively with the physics displayed 
by the cuprate superconductors and gives further support to the point of view that this $2 \mathrm{D}$ model might indeed capture several important features of these strongly-correlated materials.

Motivated also by those theoretical findings, we present in this paper a two-loop fieldtheoretical RG calculation of the renormalized couplings, the quasiparticle weight, several orderparameter response functions, and the uniform spin and charge susceptibilities of a 2D fermionic model such that only eight points located near the "hot spots" on the Fermi surface are retained, which are directly connected by spin density wave ordering wavevector. A variation of this model was recently investigated in the literature using RG methods by Abanov and Chubukov [39,40], Metlitski and Sachdev [41], and Efetov et al. [42], in which the corresponding high-energy fermions are partially integrated out in the system such that they arrive at a low-energy effective theory involving the bosonic SDW order parameter coupled to the fermionic excitations near the "hot spots". They found interesting renormalizations of several parameters of the model and also a clear breakdown of Fermi liquid behavior near these Fermi points. A similar strategy was also used recently to discuss the Ising-nematic quantum critical point in 2D metals (see, e.g., Refs. [43-45]). By contrast, our approach in the present paper follows closely the spirit of the conventional RG strategy [22], in which high-energy degrees of freedom are successively integrated out to derive an effective description in terms of renormalized low-energy fermions. This approach can be found, for instance, in the analysis performed by Furukawa and Rice [28] who first discussed a very similar model within a purely fermionic RG scheme up to one-loop order. As a consequence, these authors found, for weak Hubbard-like initial conditions, a RG flow of the renormalized interactions towards strong coupling at low energies, which seems to suggest some limitations of the one-loop RG scheme in order to have complete access to the infrared regime of this system. In our view, this fact also points to the importance of higher-order quantum corrections in the full description of the low-energy dynamics of this $2 \mathrm{D}$ model. In this respect, our present work takes seriously this observation and, for this reason, it represents a step forward in this direction.

This paper is organized as follows. In Section 2, the 2D fermionic model consisting of eight points located near the "hot spots" on the Fermi surface that we wish to study is introduced. Next (in Section 3), we explain the field-theoretical RG methodology and show how to implement this method up to two loops in order to describe this model. In this part, we will choose to present this methodology in a concise way since the field-theoretical RG scheme up to two-loop order was already described in detail by one of us in the context of another $2 \mathrm{D}$ fermionic model elsewhere [46]. The RG flow equations are derived analytically and solved numerically in Section 4 and then we proceed to discuss our main results. Lastly, Section 5 is devoted to our conclusions.

\section{Model}

We begin our analysis with a general two-dimensional energy dispersion given, for instance, by $\xi_{\mathbf{k}}=-2 t\left(\cos \left(k_{x}\right)+\cos \left(k_{y}\right)\right)-4 t^{\prime} \cos \left(k_{x}\right) \cos \left(k_{y}\right)-\mu$ with $t$ being the nearest neighbor hopping, $t^{\prime}$ the next-nearest neighbor hopping amplitude and $\mu$ the chemical potential. For the cuprates, the appropriate choice of parameters is $t^{\prime}=-0.3 t$, which results, at low hole doping, in the curved Fermi surface (FS) shown in Fig. 1. This FS intersects the antiferromagnetic zone boundary at eight points (i.e., the "hot spots"). These points are connected through Umklapp processes which leads us to examine the RG equations for all the coupling constants near these points.

The eight points around the "hot spots" are displayed in Fig. 1. If we rotate the momentum axes $\left(k_{x}, k_{y}\right)$ by $45^{\circ}$ degrees, we can define the new axes $\left(k_{\|}, k_{\perp}\right)$, where the momenta $k_{\|}$and 


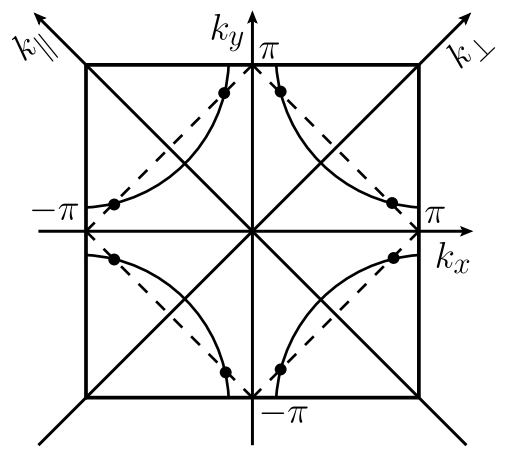

Fig. 1. The FS of the 2D fermionic model such that only eight points located near the "hot spots" on the Fermi surface are retained, which are directly connected by spin density wave ordering wavevector. The dashed-line square refers to the antiferromagnetic zone boundary in the problem.

$k_{\perp}$ refer to momentum parallel and normal to the FS, respectively. Besides, since we will be interested only in the universal quantities of this model, we shall linearize the energy dispersion around the FS as $\xi_{\mathbf{k}} \approx v_{F, \perp}\left(k_{\|}\right)\left(\left|k_{\perp}\right|-k_{F, \perp}\right)$ with the component of the Fermi velocity given by $v_{F, \perp}\left(k_{\|}\right)=\left|\nabla_{\left(k_{\|}, k_{\perp}\right)} \xi_{\mathbf{k}}\right|_{k_{\perp}=k_{F, \perp}} \mid \sin \alpha$, where $k_{F, \perp}$ is the perpendicular component of the Fermi momentum at the hot spots and $\alpha$ is the angle of the Fermi velocity on the rotated momentum axes. In terms of the parameters of the two-dimensional energy dispersion, the perpendicular component of the Fermi velocity is given by

$$
\begin{aligned}
v_{F, \perp}\left(k_{\|}\right)= & \left\{8 \sin ^{2}\left(\frac{k_{\|}}{\sqrt{2}}\right)\left[t \cos \left(\frac{k_{F, \perp}}{\sqrt{2}}\right)-t^{\prime} \cos \left(\frac{k_{\|}}{\sqrt{2}}\right)\right]^{2}\right. \\
& \left.+\left(k_{\|} \leftrightarrow k_{F, \perp}\right)\right\}^{1 / 2} \sin \alpha,
\end{aligned}
$$

and $k_{F, \perp}$ is given by

$$
k_{F, \perp}= \pm \sqrt{2} \arccos \left\{\frac{4 t \cos \left(\frac{k_{\|}}{\sqrt{2}}\right) \pm \sqrt{\Delta}}{8 t^{\prime}\left[1+\cos ^{2}\left(\frac{k_{\|}}{\sqrt{2}}\right)\right]}\right\}
$$

where $\Delta=16 t^{2} \cos ^{2}\left(k_{\|} / \sqrt{2}\right)+16 t^{\prime}\left[1+\cos ^{2}\left(k_{\|} / \sqrt{2}\right)\right]\left[\mu+4 t^{\prime} \sin ^{2}\left(k_{\|} / \sqrt{2}\right)\right]$. Both the momenta parallel to the FS and perpendicular to the FS are restricted to the interval $\left[-k_{c}, k_{c}\right]$, where $k_{c}$ essentially determines the ultraviolet (UV) momentum cutoff in our theory. This implies also an energy cutoff which is given by $\Lambda_{0}=2 v_{F} k_{c}$ which we choose to be equal to the full bandwidth of the problem, i.e. $\Lambda_{0}=8 t$.

If we use a coherent-state functional integral representation of the resulting Hamiltonian, the model at $T=0$ and constant chemical potential $\mu=E_{F}$ becomes described by the partition function $\mathcal{Z}=\int \mathcal{D}[\bar{\psi}, \psi] \exp \left(i \int_{-\infty}^{\infty} d t L[\bar{\psi}, \psi]\right)$ with the Lagrangian $L=L_{0}+L_{\text {int }}$ given by

$$
L=\sum_{\mathbf{k}, \sigma} \bar{\psi}_{\sigma}(\mathbf{k})\left(i \partial_{t}-\xi_{\mathbf{k}}\right) \psi_{\sigma}(\mathbf{k})-\sum_{i} \sum_{\substack{\mathbf{k}_{\mathbf{1}}, \mathbf{k}_{\mathbf{2}}, \mathbf{k}_{\mathbf{3}} \\ \sigma, \sigma^{\prime}}} g_{i, B} \bar{\psi}_{\sigma}\left(\mathbf{k}_{\mathbf{4}}\right) \bar{\psi}_{\sigma^{\prime}}\left(\mathbf{k}_{\mathbf{3}}\right) \psi_{\sigma^{\prime}}\left(\mathbf{k}_{\mathbf{2}}\right) \psi_{\sigma}\left(\mathbf{k}_{\mathbf{1}}\right)
$$

where $\mathbf{k}_{\mathbf{4}}=\mathbf{k}_{\mathbf{1}}+\mathbf{k}_{\mathbf{2}}-\mathbf{k}_{\mathbf{3}}$ and the volume $V$ has been set equal to unity. The Grassmann fields $\bar{\psi}_{\sigma}(\mathbf{k})$ and $\psi_{\sigma}(\mathbf{k})$ are associated, respectively, to the creation and annihilation operators of excitations lying in the vicinity of the hot spots with momentum $\mathbf{k}$ and spin projection $\sigma$. The index 

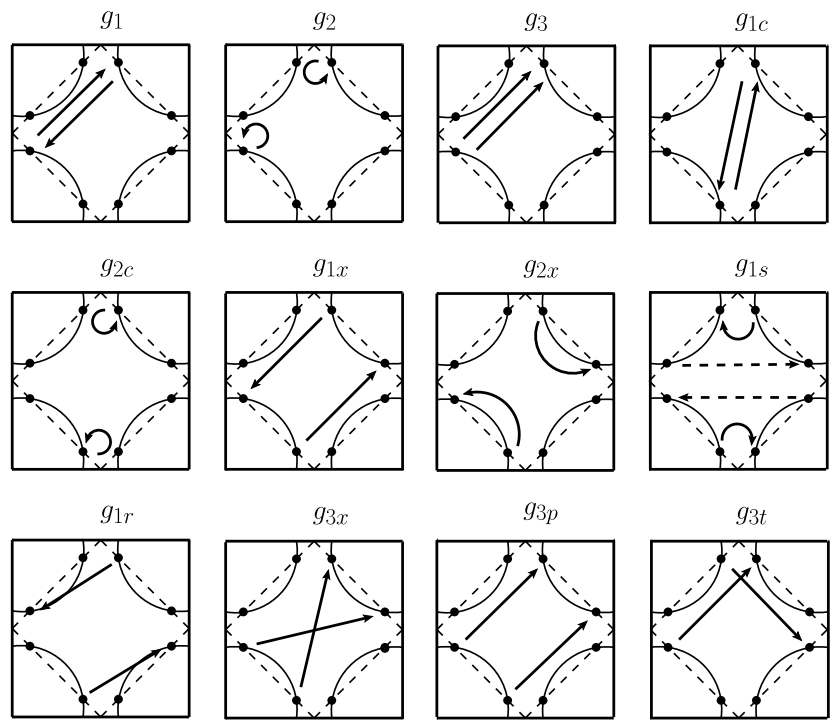

Fig. 2. The relevant couplings of the field theory model. Here we use a "g-ology" notation adapted to our 2D problem at hand. For the $g_{1 s}$-interaction, we depict two scattering processes (one by a solid line and the other by a dashed line) which are in fact always equal in our results.

$i$ runs over possible interaction processes of the model that produce logarithmic divergences within perturbation theory, i.e., $i=1,2,3,1 c, 2 c, 1 x, 2 x, 1 s, 1 r, 3 x, 3 p, 3 t$ (for details of all the couplings taken into account, see Fig. 2). In this way, in order to keep a close connection with other RG works in the literature, we are following a "g-ology" notation, adapted of course to our 2D problem at hand. The Lagrangian of Eq. (3) therefore defines our bare quantum field theory model which is regularized in the UV by the cutoff $k_{c}$ mentioned above.

\section{Two-loop RG methodology}

The methodology of our RG scheme follows closely the standard field-theoretical approach [47], which was also explained in full detail in a previous paper published by one of us [46]. If one applies a naive perturbation theory for the present model, divergences (or non-analyticities) emerge in the low-energy limit at the calculation of several important quantities of the model such as one-particle irreducible four-point vertices, self-energy, and linear response functions. This result normally implies that the bare perturbation theory setup is not appropriate for this case, since it is known to be written in terms of the microscopic parameters and not the low-energy quantities of the model. We circumvent this problem by rewriting all the bare parameters of the theory in terms of the corresponding renormalized ones plus additional counterterms. The main role of these counterterms is to regularize the theory at a floating RG scale $\Lambda$ and, for this reason, they must be calculated order by order in perturbation theory. By doing this, the newly-constructed renormalized perturbation theory becomes a well-defined expansion in terms of the renormalized couplings and, in this way, its predictions can be compared to experiments. Since this program is successfully accomplished here, the field theory model is said to be renormalizable.

At two-loop RG level, we must perform the following substitutions for the fermionic fields in the Lagrangian (Eq. (3)) of the model 


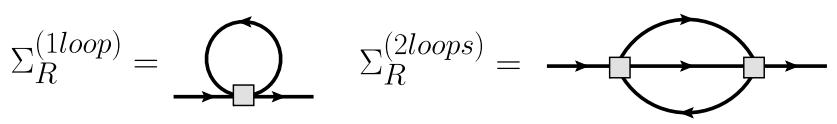

Fig. 3. Feynman diagrams for the self-energy of the model up to two-loop order. The squares represent schematically the renormalized couplings of the model. The one-loop contribution is the Hartree term and the two-loop contribution is the so-called sunset diagram.

$$
\begin{aligned}
& \psi_{\sigma}(\mathbf{k}) \rightarrow Z_{\Lambda}^{1 / 2} \psi_{R \sigma}(\mathbf{k}), \\
& \bar{\psi}_{\sigma}(\mathbf{k}) \rightarrow Z_{\Lambda}^{1 / 2} \bar{\psi}_{R \sigma}(\mathbf{k}),
\end{aligned}
$$

where $Z_{\Lambda}$ is the RG flowing quasiparticle weight which is naturally related in the limit of $\Lambda \rightarrow 0$ to the conventional many-body definition of the quasiparticle peak $Z=\left(1-\partial \operatorname{Re} \Sigma\left(\omega, \mathbf{k}=\mathbf{k}_{\mathbf{F}}\right) /\right.$ $\left.\left.\partial \omega\right|_{\omega=0}\right)^{-1}$, with $\Sigma(\omega, \mathbf{k})$ being the self-energy and $\mathbf{k}_{\mathbf{F}}$ is the Fermi vector. The corresponding Feynman diagrams up to two-loop order are shown in Fig. 3. The one-loop diagram (i.e. the Hartree term) is generally independent of the external frequency. As a result, this contribution does not renormalize the quasiparticle weight in the present case. In fact, it only generates a constant shift in the chemical potential of the model that must be appropriately subtracted by a counterterm such that the density of particles in the system remains always fixed [22]. By contrast, the two-loop contribution (i.e. the sunset diagrams) is the first contribution to the selfenergy that produces a non-analyticity as a function of the external frequency $\omega$. For this reason, this term alone will be responsible for the renormalization of the quasiparticle weight up to this order (for more details on this point, see, e.g., Refs. [46,48] in the context of different fermionic 2D models).

The so-called anomalous dimension is conventionally defined by $\eta=\Lambda d \ln Z_{\Lambda} / d \Lambda$ (see, e.g., Ref. [47]). From this expression, we obtain using a standard RG condition for the inverse of the renormalized single-particle Green's function $\Gamma_{R}^{(2)}=\left(G_{R}\right)^{-1}$, i.e. $\operatorname{Re} \Gamma_{R}^{(2)}\left(k_{0}=\Lambda, \mathbf{k}=\mathbf{k}_{\mathbf{F}}\right)=\Lambda$ that

$$
\begin{aligned}
\eta= & \frac{1}{4}\left(g_{1}^{2}+g_{2}^{2}+\frac{g_{3}^{2}}{2}+g_{1 c}^{2}+g_{2 c}^{2}+g_{1 x}^{2}+g_{2 x}^{2}+g_{3 x}^{2}\right. \\
& \left.-g_{1} g_{2}-g_{1 c} g_{2 c}-g_{1 x} g_{2 x}-g_{3 p} g_{3 x}+g_{3 p}^{2}\right) .
\end{aligned}
$$

We note that, despite the choice of a RG prescription, all flow equations up to two-loop order computed in this work are scheme-independent, i.e. they do not depend on the RG conditions used to derive these equations. In addition, we also would like to emphasize here the fact that if we were to take the 1D limit of Eq. (5), we would reproduce exactly the well-known result for the anomalous dimension for this case at two-loop order as was first calculated long ago in Ref. [49].

In addition, it can also be conventionally defined [47] that the bare and renormalized coupling constants are related to each other at two loops by

$$
g_{i, B}=N^{-1}(0) Z_{\Lambda}^{-2}\left[g_{i R}+\Delta g_{i R}^{1 \text { loop }}+\Delta g_{i R}^{2 \text { loops }}\right],
$$

where $i=1,2,3,1 c, 2 c, 1 x, 2 x, 1 s, 1 r, 3 x, 3 p, 3 t$ and the term $N(0)=k_{c} /\left(\pi^{2} v_{F, \perp}\right)$ is related to the density of states of the model at the "hot spots" points. The renormalized quantities - labeled by the subscript $R$ - generally depend on the RG scale $\Lambda$. In contrast, all the bare quantities will be denoted by the label $B$. The terms $\Delta g_{i R}^{1 \text { loop }}$ and $\Delta g_{i R}^{\text {2loops }}$ represent the counterterms necessary 


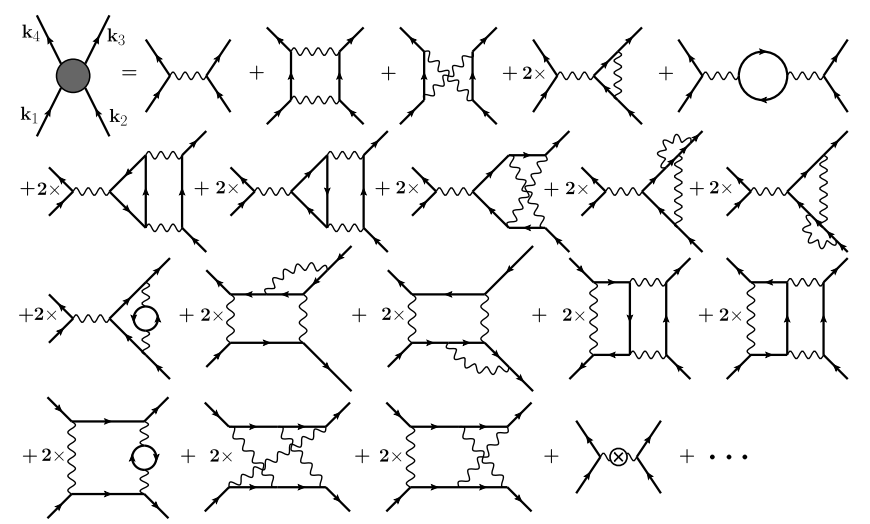

Fig. 4. Feynman diagrams for the vertex corrections up to two-loop order which yield logarithmic contributions as a function of the external energies. Solid lines denote the noninteracting fermionic single-particle Green's function, whereas the wavy lines correspond to the renormalized coupling constants. The diagram with a cross refers to the counterterm in each corresponding scattering channel. The ellipsis represents two-loop diagrams that yield log-squared contributions that are appropriately canceled by the counterterm diagrams.

to regularize at one-loop and two-loop orders, respectively, the one-particle irreducible four-point functions in each of the corresponding scattering channels.

We can now adjust these counterterms such that all divergences are exactly canceled in our series expansion for the couplings up to two loops, i.e. we choose the following standard RG condition for the one-particle irreducible four-point vertices: $\Gamma_{i, R}^{(4)}\left(k_{10}=\Lambda / 2, k_{20}=\Lambda / 2, k_{30}=\right.$ $\left.3 \Lambda / 2, k_{40}=-\Lambda / 2\right)=-i g_{i, R}(\Lambda)$, where $k_{i 0}$ are the energy components. However, the price we pay for this is the appearance of a new scale with all physical quantities now depending on this scale $\Lambda$. By contrast, the original model has no information about this quantity, i.e. the bare parameters do not depend on $\Lambda$. This leads us to the renormalization group conditions for the bare couplings of the model, i.e. $\Lambda d g_{i, B} / d \Lambda=0$. Therefore, using Eq. (6), we finally obtain

$$
\Lambda \frac{d g_{i, R}}{d \Lambda}=2 \eta g_{i, R}-\Lambda \frac{d}{d \Lambda}\left(\Delta g_{i, R}^{1 \text { loop }}+\Delta g_{i, R}^{2 \text { loops }}\right) .
$$

The initial conditions for this system of differential equations are naturally given by the microscopic interactions. The Feynman diagrams corresponding to the vertex corrections up to two-loop order are displayed in Fig. 4.

In order to investigate what are the enhanced correlations in the low-energy limit of the model, it is important to calculate the corresponding susceptibilities by introducing an infinitesimal external field in the appropriate channel and evaluating its linear response. Therefore, we must add to the Lagrangian that describes the present model the following term

$$
L_{\text {ext }}=\sum_{\mathbf{k}, \alpha, \beta} \mathcal{T}_{B, S C}^{\alpha \beta} \bar{\psi}_{\alpha}(\mathbf{k}) \bar{\psi}_{\beta}(-\mathbf{k})+\sum_{\mathbf{k}, \alpha, \beta} \mathcal{T}_{B, D W}^{\alpha \beta} \bar{\psi}_{\alpha}(\mathbf{k}+\mathbf{Q}) \psi_{\beta}(\mathbf{k})+\text { H.c. },
$$

where $\mathcal{T}_{B, S C}^{\alpha \beta}$ and $\mathcal{T}_{B, D W}^{\alpha \beta}$ are the bare response vertices for superconducting (SC) and densitywave (DW) orders, respectively, and $\mathbf{Q}=(\pi, \pi)$. This added term will generate new Feynman diagrams - the three-legged vertices displayed in Fig. 5 - which will also produce new logarithmic singularities in the low-energy limit of our field theory model (see also Refs. $[46,48]$ in the 

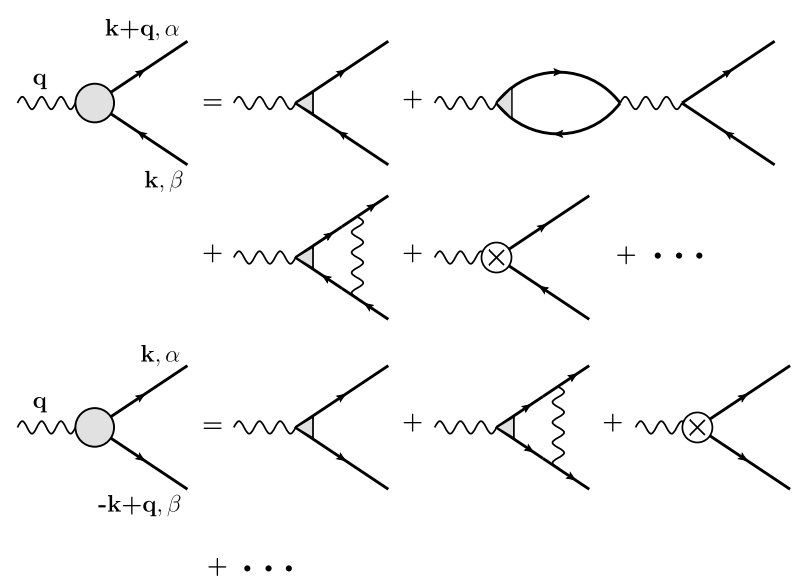

Fig. 5. Feynman diagrams for both SC and DW response functions of the model which yield logarithmic contributions as a function of the external energies. The diagrams with crosses represent the counterterms. The ellipses represent two-loop diagrams that yield log-squared contributions that are appropriately canceled by the counterterm diagrams.

context of different fermionic 2D models). Therefore, we must regularize these divergences by defining the renormalized response vertices and the corresponding counterterms as follows

$$
\begin{aligned}
& \mathcal{T}_{B, S C}^{\alpha \beta}=Z_{\Lambda}^{-1}\left[\mathcal{T}_{R, S C}^{\alpha \beta}\left(k_{\|}, k_{\perp}\right)+\Delta \mathcal{T}_{R, S C}^{\alpha \beta}\left(k_{\|}, k_{\perp}\right)\right], \\
& \mathcal{T}_{B, D W}^{\alpha \beta}=Z_{\Lambda}^{-1}\left[\mathcal{T}_{R, D W}^{\alpha \beta}\left(k_{\|}, k_{\perp}\right)+\Delta \mathcal{T}_{R, D W}^{\alpha \beta}\left(k_{\|}, k_{\perp}\right)\right] .
\end{aligned}
$$

To calculate these counterterms, we must also establish a RG prescription $\Gamma_{R, i, \alpha \beta}^{(2,1)}\left(k_{0}=\right.$ $\left.\Lambda, k_{\|}, k_{\perp}= \pm k_{F, \perp}\right)=-i \mathcal{T}_{R, i}^{\alpha \beta}\left(k_{\|}, \pm k_{F, \perp} ; \Lambda\right)$ for $i=S C$ and $D W$. As a result, by invoking the RG condition for bare quantities of the model $\Lambda\left(d \mathcal{T}_{B, S C(D W)}^{\alpha \beta} / d \Lambda\right)=0$, we obtain the flow equations for the SC response vertices

$$
\begin{aligned}
\Lambda \frac{d \mathcal{T}_{R, S C}^{(1) \alpha \beta}}{d \Lambda}= & \frac{1}{2}\left[\left(g_{2 c}+g_{1 r}\right) \mathcal{T}_{R, S C}^{(1) \alpha \beta}-\left(g_{1 c}+g_{1 r}\right) \mathcal{T}_{R, S C}^{(1) \beta \alpha}\right. \\
& \left.+\left(g_{2 x}+g_{1 s}\right) \mathcal{T}_{R, S C}^{(2) \alpha \beta}-\left(g_{1 x}+g_{1 s}\right) \mathcal{T}_{R, S C}^{(2) \beta \alpha}\right]+\eta \mathcal{T}_{R, S C}^{(1) \alpha \beta},
\end{aligned}
$$

where we defined the new vertices $\mathcal{T}_{R, S C}^{(1) \alpha \beta}=\mathcal{T}_{R, S C}^{\alpha \beta}\left(k_{\|}, k_{F, \perp}\right)$ and $\mathcal{T}_{R, S C}^{(2) \alpha \beta}=\mathcal{T}_{R, S C}^{\alpha \beta}\left(-k_{\|}, k_{F, \perp}\right)$ depending on their location at the Fermi points. As for the DW response vertices we get the following RG equations

$$
\begin{aligned}
\Lambda \frac{d \mathcal{T}_{R, D W}^{(1) \alpha \beta}}{d \Lambda}= & \frac{1}{2}\left[\left(g_{1}+g_{3}+2 g_{3 t}\right) \sum_{\sigma=\alpha, \beta} \mathcal{T}_{R, D W}^{(1) \sigma \sigma}\right. \\
& +\left(g_{1 x}+g_{3 p}+2 g_{3 t}\right) \sum_{\sigma=\alpha, \beta} \mathcal{T}_{R, D W}^{(2) \sigma \sigma}-\left(g_{3}+g_{3 x}\right) \mathcal{T}_{R, D W}^{(1) \alpha \beta} \\
& \left.-\left(g_{2}+g_{2 x}\right) \mathcal{T}_{R, D W}^{(2) \beta \alpha}\right]+\eta \mathcal{T}_{R, D W}^{(1) \alpha \beta},
\end{aligned}
$$

where we also introduced the new vertices $\mathcal{T}_{R, D W}^{(1) \alpha \beta}=\mathcal{T}_{R, D W}^{\alpha \beta}\left(k_{\|}, k_{F, \perp}\right)$ and $\mathcal{T}_{R, D W}^{(2) \alpha \beta}=$ $\mathcal{T}_{R, D W}^{\alpha \beta}\left(-k_{\|}, k_{F, \perp}\right)$ according to their association to the hot spots. By symmetrizing and 
antisymmetrizing all the response vertices w.r.t. the spin indices, we obtain the following order parameters

$$
\left\{\begin{aligned}
& \mathcal{T}_{S S C}^{(j)}= \mathcal{T}_{R, S C}^{(j) \uparrow \downarrow}-\mathcal{T}_{R, S C}^{(j) \downarrow \uparrow}, \\
& \mathcal{T}_{T S C}^{(j)}=\left\{\begin{array}{l}
\mathcal{T}_{R, S C}^{(j) \uparrow \uparrow} \quad\left(S_{z}=1\right), \\
\mathcal{T}_{R, S C}^{(j) \uparrow \downarrow}+\mathcal{T}_{R, S C}^{(j) \downarrow \uparrow} \quad\left(S_{z}=0\right),, \\
\mathcal{T}_{R, S C}^{(j) \downarrow \downarrow} \quad\left(S_{z}=-1\right)
\end{array}\right. \\
& \mathcal{T}_{C D W}^{(j)}=\mathcal{T}_{R, D W}^{(j) \uparrow \uparrow}+\mathcal{T}_{R, D W}^{(j) \downarrow \downarrow}, \\
& \mathcal{T}_{S D W}^{(j)}=\mathcal{T}_{R, D W}^{(j) \uparrow \uparrow}-\mathcal{T}_{R, D W}^{(j) \downarrow \downarrow},
\end{aligned}\right.
$$

where $j=1,2$ and the subscripts SSC and TSC correspond to singlet and triplet superconductivity, whereas $S D W$ and $C D W$ stand for charge and spin-density waves, respectively. Hence, using the above relations one can derive in a straightforward way the RG flow equations for each response vertex associated with a potential instability of the normal state toward a given ordered (i.e. symmetry-broken) phase. In order to determine the symmetry of the order parameter we must further symmetrize the response vertices w.r.t. the index $j$. Thus

$$
\left\{\begin{array}{l}
\mathcal{T}_{S S C}^{(s-\text { wave })}=\mathcal{T}_{S S C}^{(1)}+\mathcal{T}_{S S C}^{(2)} \\
\mathcal{T}_{S S C}^{(d \text {-wave })}=\mathcal{T}_{S S C}^{(1)}-\mathcal{T}_{S S C}^{(2)} \\
\mathcal{T}_{T S C}^{(p \text {-wave })}=\mathcal{T}_{T S C}^{(1)}+\mathcal{T}_{T S C}^{(2)} \\
\mathcal{T}_{C D W}=\mathcal{T}_{C D W}^{(1)}+\mathcal{T}_{C D W}^{(2)} \\
\mathcal{T}_{S D W}=\mathcal{T}_{S D W}^{(1)}+\mathcal{T}_{S D W}^{(2)} \\
\mathcal{T}_{C F}=\mathcal{T}_{C D W}^{(1)}-\mathcal{T}_{C D W}^{(2)} \\
\mathcal{T}_{S F}=\mathcal{T}_{S D W}^{(1)}-\mathcal{T}_{S D W}^{(2)}
\end{array}\right.
$$

Here the labels $s$-wave, $d$-wave and $p$-wave refer to the symmetries of the corresponding SC order parameters, and $C F$ and $S F$ stand for charge and spin flux phases, respectively.

We now move on to discuss the uniform $(\mathbf{q} \rightarrow 0)$ linear response functions of the model. At one-loop level, we find that there are no Feynman diagrams that produce logarithmic singularities in the low-energy limit. In this way, it becomes necessary to go up to two loops in order to obtain the appropriate RG equations for this quantity (see Fig. 6). To do this, we must add new bare auxiliary fields $\mathcal{T}_{B}^{\alpha \alpha}(\mathbf{q} \approx \mathbf{0})$ that couple, respectively, to the charge and spin densities in the Lagrangian of the model as follows

$$
L_{\text {ext }}^{\prime}=\sum_{\mathbf{k}}\left[\mathcal{T}_{B}^{\uparrow \uparrow}(\mathbf{q}) \bar{\psi}_{\uparrow}(\mathbf{k}) \psi_{\uparrow}(\mathbf{k})+\mathcal{T}_{B}^{\downarrow \downarrow}(\mathbf{q}) \bar{\psi}_{\downarrow}(\mathbf{k}) \psi_{\downarrow}(\mathbf{k})\right]
$$

Thus, we must regularize these divergences by defining the renormalized uniform response functions and the corresponding counterterms as follows [50,51]

$$
\mathcal{T}_{B}^{\alpha \alpha}(\mathbf{q})=Z_{\Lambda}^{-1}\left[\mathcal{T}_{R}^{\alpha \alpha}(\mathbf{q})+\Delta \mathcal{T}_{R}^{\alpha \alpha}(\mathbf{q})\right]
$$

where the $Z_{\Lambda}$ factor originates, as discussed previously, from the redefinition of the fermionic fields at two-loop RG level displayed in Eq. (4) and is naturally related to the self-energy feedback into the equations. In order to determine these counterterms, we must change the RG 


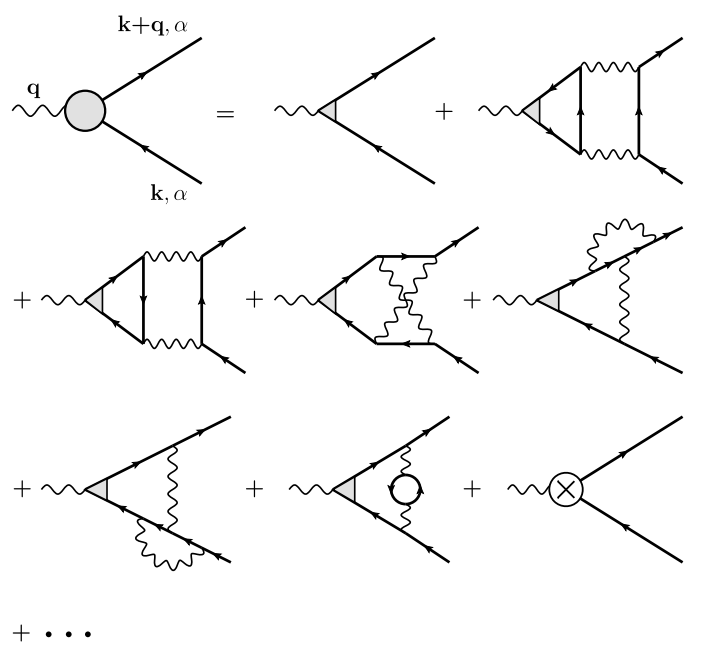

Fig. 6. Feynman diagrams for uniform $(\mathbf{q} \rightarrow 0)$ response functions of the model up to two loops which yield logarithmic contributions as a function of the normal component of the external momentum. Since the corresponding one-loop diagrams for this case are non-divergent near the hot spots, they turn out not to be relevant in this calculation. The diagram with a cross stands for the counterterm. The ellipsis represents higher-loop diagrams that are appropriately canceled by the counterterm diagrams.

condition of the three-legged vertices to $\Gamma_{R, \alpha \alpha}^{(2,1)}\left(k_{0}=0, v_{F, \perp}\left(k_{\|}\right)\left(\left|k_{\perp}\right|-k_{F, \perp}\right)=\Lambda, \mathbf{q} \approx 0\right)=$ $-i \mathcal{T}_{R}^{\alpha \alpha}(\mathbf{q} ; \Lambda)$ since we will now approach the low-energy limit of the model via the momentum scale. Following this prescription, by establishing the RG condition for the bare quantities of the model $\Lambda\left(d \mathcal{T}_{B}^{\alpha \alpha} / d \Lambda\right)=0$, we obtain the RG equations for the renormalized uniform response functions

$$
\Lambda \frac{d \mathcal{T}_{R}^{\alpha \alpha}(\mathbf{q})}{d \Lambda}=\eta \mathcal{T}_{R}^{\alpha \alpha}(\mathbf{q})-\Lambda \frac{d \Delta \mathcal{T}_{R}^{\alpha \alpha}(\mathbf{q})}{d \Lambda} .
$$

By symmetrizing and antisymmetrizing these quantities w.r.t. spin indices, i.e.

$$
\left\{\begin{array}{l}
\mathcal{T}_{C}=\mathcal{T}_{R}^{\uparrow \uparrow}+\mathcal{T}_{R}^{\downarrow \downarrow} \\
\mathcal{T}_{S}=\mathcal{T}_{R}^{\uparrow \uparrow}-\mathcal{T}_{R}^{\downarrow \downarrow}
\end{array}\right.
$$

we readily obtain the corresponding flow equations for both renormalized charge $\left(\mathcal{T}_{C}\right)$ and spin $\left(\mathcal{T}_{S}\right)$ uniform response functions.

Lastly, once we computed the response vertices associated with all the order parameters, we can proceed to calculate their corresponding renormalized susceptibilities. They are given by

$$
\chi_{m}(\Lambda)=N(0) \int_{0}^{l} d \xi \mathcal{T}_{m}(\xi) \mathcal{T}_{m}^{*}(\xi)
$$

where $m=S S C$ (s-wave), SSC(d-wave), TSC ( $p$-wave), CDW, SDW, $C F$ and $S F$, and the RG step $l$ is defined by $l=\ln \left(\Lambda_{0} / \Lambda\right)$. As for the uniform susceptibilities, one obtains that $\chi_{n}(\Lambda)=$ $N(0)\left|\mathcal{T}_{n}(\Lambda)\right|^{2}$, where $n=C$ and $S$. 


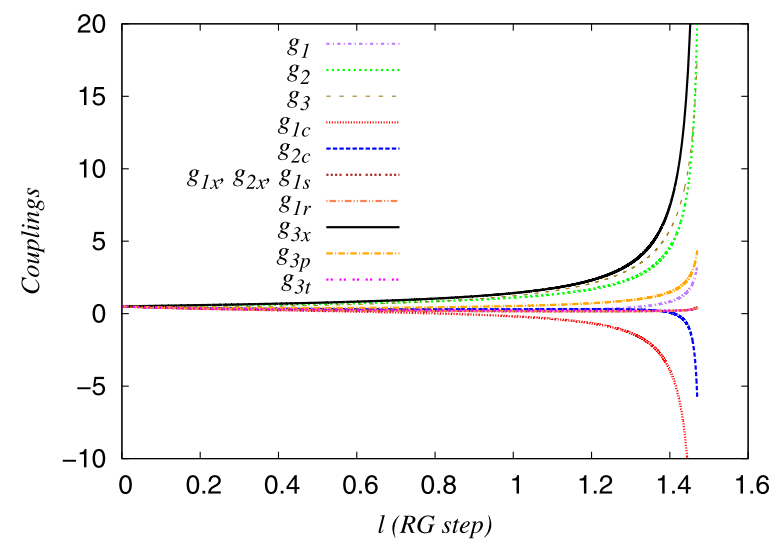

Fig. 7. (Color online.) One-loop RG flow for all the coupling constants of the model for the initial choice of $g_{i}^{(0)}=0.5$.

\section{Two-loop RG flow equations and numerical solution}

In this section, we discuss the RG flow equations up to two-loop order associated with all the physical quantities calculated previously in this paper and show their numerical solution as one approaches the low-energy limit of the model. Concerning the renormalized couplings, we choose to write down for completeness all the RG equations up to two-loop order explicitly in Appendix A. It is important to stress here that, up to one-loop order, our equations agree with those derived by Furukawa and Rice (Ref. [28]) who first discussed a very similar model within a one-loop RG approximation in the literature. However, as we have explained previously in this paper, to go beyond the work in Ref. [28] we need to investigate also the effect of higher-order quantum fluctuations in order to describe their impact on the low-energy dynamics of the system.

To solve all the coupled differential RG equations numerically, we apply standard fourth-order Runge-Kutta method. As an initial condition for these equations, we take Hubbard-like repulsive parameters, i.e. $g_{i}^{(0)}=\left[k_{c} /\left(\pi^{2} v_{F, \perp}\right)\right] U$ for all couplings. For simplicity, we will always choose $g_{i}^{(0)}=0.5$ in the present work. Using the experimental data from Ref. [3], since the nodal liquid is stabilized for $4 \%$ of hole doping, this implies that $v_{F, \perp} \approx 1.70 t$ at the "hot spots" and the Hubbard local interaction parameter $U \approx 3.6 t$. We mention here, however, that our results do not depend crucially on this initial choice of the coupling constants and we emphasize that they are robust within an appreciable range of these physical parameters.

In order to establish a direct comparison between the one-loop RG results and the two-loop RG data for this 2D model, we first present a plot of the flow of the couplings up to one-loop order only. As a result, the corresponding one-loop RG flow as a function of the step $l$ is displayed in Fig. 7. In agreement with Ref. [28], we confirm that despite the fact that the couplings are initially taken to be weak, the one-loop RG flow is always to a strong coupling regime in the low-energy limit for Hubbard-like initial interactions. Additionally, we observe that many renormalized couplings tend to diverge as the RG scale is lowered at the same critical scale $l_{c}=\ln \left(\Lambda_{0} / \Lambda_{c}\right)$, after which point the RG approach up to one loop clearly breaks down. This fact seems to limit the validity of the one-loop RG calculation in order to study the infrared regime of this model and invites one to go beyond that approximation in order to access the low-energy limit of the system. 


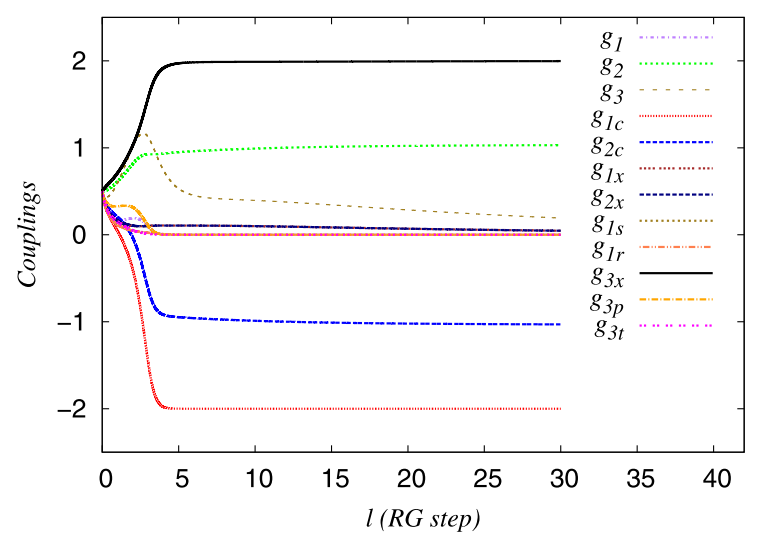

Fig. 8. (Color online.) Two-loop RG flow for all the coupling constants of the model for the initial choice of $g_{i}^{(0)}=0.5$.

Following this strategy, we now move on to our two-loop RG results. The RG flow up to two-loop order for the couplings as a function of the step $l$ is depicted in Fig. 8. In this plot, we observe that instead of displaying a divergent behavior as shown in the one-loop RG flow, all renormalized couplings at two-loop RG level now clearly approach asymptotically infrared stable fixed points in the low-energy limit. It is true, however, that some couplings become saturated at reasonably strong coupling fixed points. This is a well-known problem in RG theory and happens as well in other applications of this method to quantum field theories in which fluctuation effects are known to be strong (the most notorious example being the Wilson-Fisher fixed point in $\phi^{4}$-theory in three dimensions [52,53]). Notwithstanding this fact, we point out that, quite surprisingly, the two-loop RG approach turns out to yield a better controlled theory for the present model than the one-loop RG scheme and, for this reason, we can hope that it could describe at least qualitatively the correct trend of the low-energy dynamics of the system. Two increasingly relevant renormalized couplings in the RG flow turn out to be the Umklapp scattering $g_{3 x}$ and the forward scattering $g_{2}$. As we will see shortly, they both tend to favor SDW antiferromagnetic ordering tendencies in the system. By contrast, upon inclusion of quantum fluctuation effects, the Cooper pair interaction processes $g_{1 c}$ and $g_{2 c}$ - that are initially taken to be repulsive - flow naturally to attractive infrared stable fixed points in the low-energy limit. These latter couplings in turn enhance SC pairing correlations which will of course tend to manifest itself as a possible competing order in the system. As can also be inferred from Fig. 8, the remaining couplings flow asymptotically to zero in the infrared regime and, as a result, they become (dangerously) irrelevant in the low-energy effective description of this $2 \mathrm{D}$ model.

Next, we focus on the RG flow equation up to two-loop order for the quasiparticle weight $Z_{\Lambda}$ of the model (see Eq. (5)). By solving this equation numerically with initial condition $Z_{\Lambda}(l=0)=1$, we obtain the results depicted in the plot of Fig. 9 as a function of the RG step $l$. We observe that this quantity becomes universally suppressed as a power-law $Z_{\Lambda} \sim\left(\Lambda / \Lambda_{0}\right)^{\eta}$ with $\eta \approx 2.01$ in the low-energy limit, indicating a complete absence of coherent quasiparticles and a clear breakdown of Fermi liquid behavior near the "hot spots" for this regime. This conclusion is also in qualitative agreement with other works available in the literature [39-41]. Thus, in order to investigate the true nature of this low-energy state, we must further characterize the model by examining what are the dominant fluctuations that drive the system to a nullified $Z_{\Lambda}$ in the low-energy limit. 


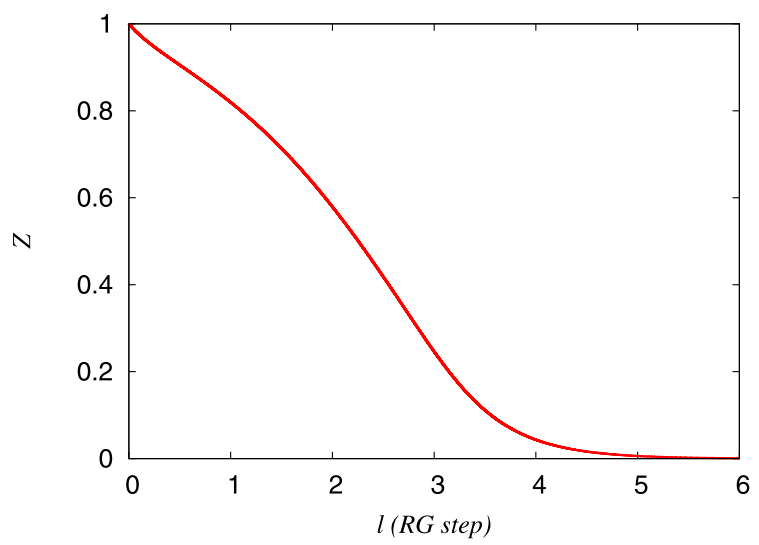

Fig. 9. (Color online.) Two-loop RG flow for the quasiparticle weight $Z_{\Lambda}$ of the model for the initial choice of $g_{i}^{(0)}=0.5$.

For this reason, we now turn our attention to the behavior of the various order-parameter susceptibilities as a function of the RG scale $\Lambda$ of the model. Using Eqs. (10) and (11), we write down explicitly the corresponding RG flow equations for these quantities up to two-loop order as follows

$$
\begin{aligned}
& \dot{\mathcal{T}}_{S S C}^{\text {s-wave }}=\frac{1}{2}\left(g_{2 c}+g_{1 c}+g_{1 x}+g_{2 x}+2 g_{1 s}+2 g_{1 r}\right) \mathcal{T}_{\text {SSC }}^{\text {s-wave }}+\eta \mathcal{T}_{\text {SSC }}^{\text {s-wave }}, \\
& \dot{\mathcal{T}}_{S S C}^{\text {-wave }}=\frac{1}{2}\left(g_{2 c}+g_{1 c}-g_{1 x}-g_{2 x}-2 g_{1 s}+2 g_{1 r}\right) \mathcal{T}_{S S C}^{d \text {-wave }}+\eta \mathcal{T}_{S S C}^{d \text {-wave }}, \\
& \dot{\mathcal{T}}_{\text {TSC }}^{\text {p-wave }}=\frac{1}{2}\left(g_{2 c}-g_{1 c}+g_{2 x}-g_{1 x}\right) \mathcal{T}_{T S C}^{\text {p-wave }}+\eta \mathcal{T}_{T S C}^{\text {p-wave }}, \\
& \dot{\mathcal{T}}_{C D W}=\frac{1}{2}\left(2 g_{1}+g_{3}+2 g_{1 x}+2 g_{3 p}+8 g_{3 t}-g_{3 x}-g_{2}-g_{2 x}\right) \mathcal{T}_{C D W}+\eta \mathcal{T}_{C D W}, \\
& \dot{\mathcal{T}}_{S D W}=-\frac{1}{2}\left(g_{2}+g_{2 x}+g_{3}+g_{3 x}\right) \mathcal{T}_{S D W}+\eta \mathcal{T}_{S D W}, \\
& \dot{\mathcal{T}}_{C F}=\frac{1}{2}\left(2 g_{1}+g_{2}-2 g_{1 x}+g_{2 x}+g_{3}-2 g_{3 p}-g_{3 x}\right) \mathcal{T}_{C F}+\eta \mathcal{T}_{C F}, \\
& \dot{\mathcal{T}}_{S F}=-\frac{1}{2}\left(g_{3}+g_{3 x}-g_{2}-g_{2 x}\right) \mathcal{T}_{S F}+\eta \mathcal{T}_{S F},
\end{aligned}
$$

where we are using the shorthand notation $\dot{\mathcal{T}}_{i}=\Lambda\left(d \mathcal{T}_{i}\right) /(d \Lambda)$. We solve these differential equations using the same numerical procedure explained above and, by using Eq. (15), we are able to follow the RG flow of the corresponding susceptibilities as a function of the RG step $l$. The results are plotted in Fig. 10. In this figure, we obtain that even though many susceptibilities are renormalized due to interactions, they remain non-divergent and become saturated at plateaus in the low-energy limit at two loops. In other words, the self-energy feedback and the higher-order vertex corrections at two-loop RG level are sufficient to suppress the divergence of these physical quantities which would show up in one-loop RG calculations. Our present two-loop RG result implies that there should be no spontaneous symmetry breaking associated with these renormalized order-parameter susceptibilities investigated here in this work and therefore the system is not expected to exhibit long-range order in any of these channels at low energies. Indeed, despite the fact that the leading short-range correlations - by at least two orders of magnitude - for this 


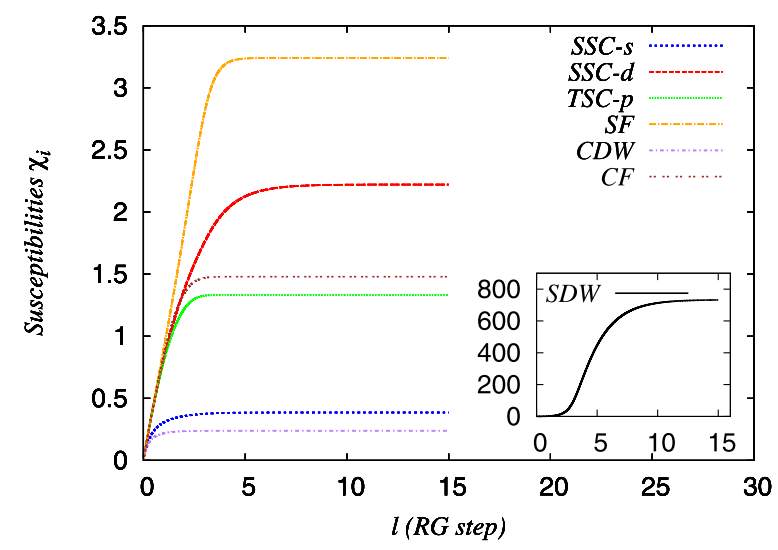

Fig. 10. (Color online.) Two-loop RG flow for various order-parameter susceptibilities of the model (in units of $N(0)$ ) for the initial choice of $g_{i}^{(0)}=0.5$.

regime are of SDW-type (i.e. antiferromagnetic spin correlations), spin flux phase fluctuations also appear as a subleading ordering tendency and $d$-wave singlet SC clearly comes in third place. This suggests that a possible coexistence of such short-ranged ordering tendencies could be the hallmark of the true nature of the corresponding low-energy state.

Other physical quantities which are naturally of high importance to potentially identify the nature of the ground state of the model are the uniform $(\mathbf{q} \rightarrow 0)$ susceptibilities in both spin and charge sector. After a lengthy but straightforward calculation, we obtain that the resulting RG flow equations for the uniform response functions up to two-loop order are the following

$$
\begin{aligned}
& \dot{\mathcal{T}}_{S}=\frac{1}{2}\left[g_{1}^{2}+g_{1 x}^{2}+g_{1 c}^{2}+\left(g_{3 p}-g_{3 x}\right)^{2}\right] \mathcal{T}_{S}, \\
& \dot{\mathcal{T}}_{C}=\frac{1}{2}\left[g_{3}^{2}+g_{3 p}^{2}+g_{3 x}^{2}+\left(g_{3 p}-g_{3 x}\right)^{2}\right] \mathcal{T}_{C},
\end{aligned}
$$

where $\dot{\mathcal{T}}_{i}=\Lambda\left(d \mathcal{T}_{i}\right) /(d \Lambda)$. We emphasize here the fact that if we take, as an important check, the $1 \mathrm{D}$ limit of the above two-loop RG equations for the uniform spin and charge response functions, we are able to reproduce established results available in the literature for these systems in various regimes (see, e.g., Refs. [49,54,55]).

In the present 2D model, we once more follow the numerical procedure described before in order to solve the equations given by Eqs. (23) and Eq. (15). Our result for the uniform charge susceptibility is shown in Fig. 11. We observe from this plot that, for repulsive Hubbard-like initial conditions, the uniform charge susceptibility (or simply charge compressibility) becomes strongly suppressed and renormalizes to zero in the low-energy limit. This implies that there should be a gap opening in the charge excitation spectrum in the system. This charge gap is produced by the Umklapp coupling parameter $g_{3 x}$, which, as we have seen before, becomes increasingly relevant in the low-energy effective theory. At this point, we draw attention to the fact that our result bears some resemblance to the well-studied case of the two-chain repulsive Hubbard ladder exactly at half-filling [56,57], where it is by now well established that the mechanism for charge gap generation in that system is driven by Umklapp interaction in the scaling limit. Therefore, we may conclude from this that the nature of the low-energy state of the present 


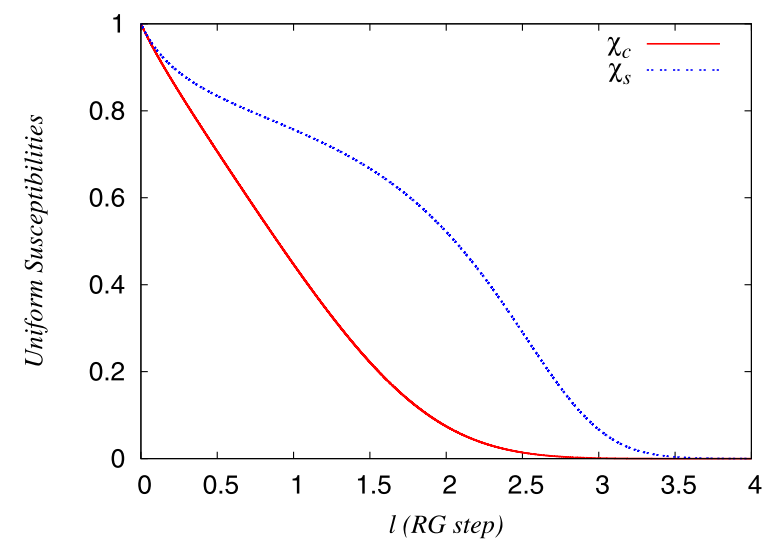

Fig. 11. (Color online.) Two-loop RG flow for both charge (C) and spin (S) uniform susceptibilities of the model (in units of $N(0))$ for the initial choice of $g_{i}^{(0)}=0.5$.

2D model is clearly insulating, which also agrees with our previous result that the quasiparticle weight $Z_{\Lambda}$ is nullified in the low-energy limit.

In Fig. 11, we also display the RG flow for the uniform spin susceptibility of the system for repulsive Hubbard-like initial conditions. As a result, we observe from this plot that even though this quantity is initially less suppressed in the RG flow than the charge compressibility, it also scales down eventually to zero in the low-energy limit. This indicates that there should be also a gap in the spin excitation spectrum of the model. The opening of a spin gap is produced by an interplay between the Umklapp coupling parameter $g_{3 x}$ (which also promotes SDW antiferromagnetic fluctuations and leads to charge gap formation) and the Cooper pair interaction process $g_{1 c}$, which in turn renormalizes to an attractive infrared stable fixed point at low energies and enhances $d$-wave pairing correlations. This result strongly hints at pronounced but short-ranged antiferromagnetic spin correlations coexisting with short-ranged $d$-wave singlet superconductivity as the underlying physics of this corresponding low-energy state.

Hence, it seems reasonable to suppose that our two-loop RG data could suggest that the lowenergy state of the present 2D model may indeed share some similarities to the $d$-Mott phase of the half-filled two-chain repulsive Hubbard ladder where the spin gap is well-understood to be related to a precursor effect of "pre-formed" Cooper pairs with approximate $d$-wave symmetry in an otherwise insulating system [56,57]. This interpretation is in line with Ref. [28], where the authors also concluded, based on a one-loop RG calculation, that the low-energy state of this 2D model should be given by an insulating spin-gapped state. However, we point out here that, within a RG computation up to one-loop order only, one cannot be sure about the spin properties of this state since the uniform spin susceptibility and the SDW spin susceptibility actually behave in a contradictory manner [28]. Our present two-loop RG results resolve this contradiction in a most natural way. Since the SDW susceptibility at two-loop RG level becomes saturated at a plateau at low energies, it cannot be associated to any long-range spin order. This is also consistent with the fact that the low-energy state naturally displays a spin gap. Therefore, we conclude that such an insulating spin-gapped state could be produced by quantum disordering effects induced by correlations included in the present 2D model, and the universal properties of this state are captured qualitatively by our two-loop RG scheme within a weak-to-moderate coupling regime. 


\section{Conclusions}

We have performed a RG calculation of a 2D model consisting of eight points located near the "hot spots" on a Fermi surface which are directly connected by SDW ordering wave vector. By following the field-theoretical RG strategy, we have derived the corresponding flow equations for the couplings, the quasiparticle weight, the order-parameter susceptibilities and the uniform spin and charge susceptibilities of the model up to two-loop order. As a result, we have found for physically relevant choices of parameters that while SDW antiferromagnetism, spin flux phase, and $d$-wave superconductivity manifest themselves as coexisting short-range ordering tendencies, the quasiparticle weight $Z_{\Lambda}$ renormalizes to zero in the low-energy limit, indicating the breakdown of the Fermi liquid behavior for this regime. Moreover, both uniform spin and charge susceptibilities scale down to zero in the same limit, which point to the fact that gap openings should take place in both spin and charge excitation spectra of the model. By comparing those results with other well-studied systems such as the half-filled two-chain repulsive Hubbard ladder, we have shown that our two-loop RG data are consistent with an interpretation that the low-energy state of the present 2D model should be given by an insulating spin-gapped state displaying no long-range antiferromagnetic order at zero temperature.

On the other hand, we would like to emphasize here that, despite the fact that the present 2D model might have a few analogies with the well-known physics of the pseudogap phase of the cuprate superconductors, this model must be of course extended in order to describe the complete Fermi surface (and, consequently, its process of either becoming partly truncated or fully reconstructed in momentum space) displayed by these compounds. For this reason, we believe that the present two-loop RG result could potentially give some insights to this problem from a weak-to-moderate coupling perspective. Lastly, we point out that it would be naturally very interesting to perform such a complete two-loop RG analysis to discuss the fully $2 \mathrm{D} t-t^{\prime}$ Hubbard model on a square lattice with a choice of parameters that precisely match the Fermi surface observed experimentally in these materials. This will introduce some expected complications such as the substantial increase of the renormalized couplings as they become in this case functions of three independent momenta of the interacting particles at low energies, but this can be handled numerically using standard techniques. Therefore, we plan to perform such a RG investigation of the $2 \mathrm{D} t-t^{\prime}$ Hubbard model in another publication.

\section{Acknowledgements}

We acknowledge financial support from CNPq and FAPEG for this project.

\section{Appendix A}

In this appendix, we show explicitly the RG flow equations up to two loops for the model. They read

$$
\begin{aligned}
\Lambda \frac{d g_{1}}{d \Lambda}= & g_{1}^{2}+g_{1 x}^{2}+4 g_{3 t}^{2}+g_{3 p}^{2}-g_{1 x} g_{2 x}-g_{3 p} g_{3 x} \\
& +\frac{1}{2}\left(g_{1 x} g_{2 x}-g_{2 x}^{2}-g_{3 p} g_{3 x}\right) g_{1 c} \\
& +\frac{1}{2}\left(g_{1 c}^{2}+g_{1}^{2}+g_{1 x}^{2}+g_{2 x}^{2}-g_{1 x} g_{2 x}-g_{3 p} g_{3 x}+g_{3 p}^{2}+g_{3 x}^{2}\right) g_{1},
\end{aligned}
$$




$$
\begin{aligned}
& \Lambda \frac{d g_{2}}{d \Lambda}=\frac{1}{2}\left(g_{1}^{2}-g_{2 x}^{2}-g_{3}^{2}-g_{3 x}^{2}\right)+\frac{1}{4}\left(g_{1}^{3}+g_{1 c} g_{1 x}^{2}+g_{1} g_{1 c}^{2}\right)+\frac{1}{4}\left(2 g_{2}-g_{1}\right) g_{3}^{2} \\
& +\frac{1}{4}\left[\left(2 g_{2 c}-g_{1 c}\right)\left(g_{3 p}^{2}+g_{3 x}^{2}\right)-2 g_{2 c} g_{3 p} g_{3 x}+2 g_{2}\left(g_{3 p}^{2}+g_{3 x}^{2}-g_{3 p} g_{3 x}\right)\right] \\
& +\frac{1}{2}\left(g_{2}-g_{2 c}\right)\left(g_{1 x}^{2}+g_{2 x}^{2}-g_{1 x} g_{2 x}\right) \text {, } \\
& \Lambda \frac{d g_{3}}{d \Lambda}=\left(g_{1}-2 g_{2}\right) g_{3}+4 g_{3 t}^{2}-g_{1 x}\left(g_{3 x}-2 g_{3 p}\right)-g_{2 x}\left(g_{3 p}+g_{3 x}\right) \\
& +\frac{1}{4}\left[\left(g_{1}-2 g_{2}\right)^{2}+\left(g_{1 c}-2 g_{2 c}\right)^{2}+2 g_{1 x}^{2}+2 g_{2 x}^{2}-2 g_{1 x} g_{2 x}\right. \\
& \left.-2 g_{3 p} g_{3 x}+2 g_{3 p}^{2}+2 g_{3 x}^{2}+g_{3}^{2}\right] g_{3}, \\
& \Lambda \frac{d g_{1 c}}{d \Lambda}=g_{1 c}^{2}+g_{1 x} g_{2 x}+g_{1 s}^{2}+g_{1 r}^{2}+g_{3 x}^{2}-g_{3 p} g_{3 x}+\frac{1}{2}\left(g_{1 x} g_{2 x}-g_{2 x}^{2}-g_{3 p} g_{3 x}\right) g_{1} \\
& +\frac{1}{2}\left(g_{1 c}^{2}+g_{1}^{2}+g_{1 x}^{2}+g_{2 x}^{2}-g_{1 x} g_{2 x}+g_{3 p}^{2}+g_{3 x}^{2}-g_{3 p} g_{3 x}\right) g_{1 c}, \\
& \Lambda \frac{d g_{2 c}}{d \Lambda}=\frac{1}{2}\left(g_{1 c}^{2}+g_{1 x}^{2}+g_{2 x}^{2}+2 g_{1 s}^{2}+2 g_{1 r}^{2}-g_{3 p}^{2}\right)+\frac{1}{4}\left(g_{1} g_{1 x}^{2}+g_{1 c} g_{1}^{2}+g_{1 c}^{3}\right) \\
& +\frac{1}{2}\left(g_{2 c}-g_{2}\right)\left(g_{1 x}^{2}+g_{2 x}^{2}-g_{1 x} g_{2 x}\right)+\frac{1}{4}\left(2 g_{2 c}-g_{1 c}\right) g_{3}^{2} \\
& +\frac{1}{4}\left[\left(2 g_{2}-g_{1}\right)\left(g_{3 p}^{2}+g_{3 x}^{2}\right)-2 g_{2} g_{3 p} g_{3 x}+2 g_{2 c}\left(g_{3 p}^{2}+g_{3 x}^{2}-g_{3 p} g_{3 x}\right)\right], \\
& \Lambda \frac{d g_{1 x}}{d \Lambda}=g_{1 c} g_{2 x}+g_{2 c} g_{1 x}+2 g_{1 s} g_{1 r} \\
& +\frac{1}{2}\left(g_{1} g_{2 c}+g_{1 c} g_{2}-2 g_{2 c} g_{2}-\frac{g_{3 p}^{2}}{2}-\frac{g_{3 x}^{2}}{2}\right) g_{1 x}+2 \eta g_{1 x}, \\
& \Lambda \frac{d g_{2 x}}{d \Lambda}=g_{1 c} g_{1 x}+g_{2 c} g_{2 x}+2 g_{1 s} g_{1 r}+\frac{1}{2}\left(g_{1 c} g_{1} g_{1 x}-2 g_{1 c} g_{1} g_{2 x}+g_{1 c} g_{2} g_{2 x}\right. \\
& \left.+g_{1} g_{2 c} g_{2 x}-2 g_{2 c} g_{2} g_{2 x}-g_{1 x} g_{3 p} g_{3 x}+g_{2 x} g_{3 p} g_{3 x}-\frac{1}{2} g_{2 x}\left(g_{3 x}^{2}+g_{3 p}^{2}\right)\right) \\
& +2 \eta g_{2 x} \\
& \Lambda \frac{d g_{1 s}}{d \Lambda}=\left(g_{1 c}+g_{2 c}\right) g_{1 s}+\left(g_{1 x}+g_{2 x}\right) g_{1 r}+2 \eta g_{1 s}, \\
& \Lambda \frac{d g_{1 r}}{d \Lambda}=\left(g_{1 c}+g_{2 c}\right) g_{1 r}+\left(g_{1 x}+g_{2 x}\right) g_{1 s}+2 \eta g_{1 r}, \\
& \Lambda \frac{d g_{3 p}}{d \Lambda}=\left(2 g_{1}-g_{2 c}\right) g_{3 p}+g_{1 x} g_{3}+4 g_{3 t}^{2}-g_{2} g_{3 p}-g_{2 x} g_{3}-g_{1} g_{3 x} \\
& +\frac{1}{2}\left(2 g_{2 c} g_{2} g_{3 p}+g_{2 x}^{2} g_{3 x}-g_{1} g_{2 c} g_{3 p}-g_{1 c} g_{2} g_{3 p}-g_{1 x} g_{2 x} g_{3 x}\right. \\
& \left.-g_{1 c} g_{1} g_{3 x}-\frac{g_{1 x}^{2} g_{3 p}}{2}\right)+2 \eta g_{3 p},
\end{aligned}
$$




$$
\begin{aligned}
\Lambda \frac{d g_{3 x}}{d \Lambda}= & \left(2 g_{1 c}-g_{2 c}\right) g_{3 x}-g_{1 c} g_{3 p}-g_{2 x} g_{3}-g_{2} g_{3 x} \\
& +\frac{1}{2}\left(2 g_{2 c} g_{2} g_{3 x}+g_{2 x}^{2} g_{3 p}-g_{1} g_{2 c} g_{3 x}-g_{1 c} g_{2} g_{3 x}-g_{1 x} g_{2 x} g_{3 p}\right. \\
& \left.-g_{1 c} g_{1} g_{3 p}-\frac{g_{1 x}^{2} g_{3 x}}{2}\right)+2 \eta g_{3 x}, \\
\Lambda \frac{d g_{3 t}}{d \Lambda}= & \left(2 g_{1}-g_{2}+g_{3}+2 g_{1 x}-g_{2 x}+2 g_{3 p}-g_{3 x}\right) g_{3 t}+2 \eta g_{3 t},
\end{aligned}
$$

where $\Lambda$ is the corresponding $\mathrm{RG}$ flowing scale and $\eta$ is naturally the anomalous dimension defined in Eq. (5). As we have discussed previously in this paper, the anomalous dimension is related to the self-energy feedback into the two-loop RG flow equations.

\section{References}

[1] For a review, see P.A. Lee, N. Nagaosa, X.-G. Wen, Rev. Mod. Phys. 78 (2006) 17.

[2] P.W. Anderson, Science 235 (1987) 1196.

[3] U. Chatterjee, M. Shi, D. Ai, J. Zhao, A. Kanigel, S. Rosenkranz, H. Raffy, Z.Z. Li, K. Kadowaki, D.G. Hinks, Z.J. Xu, J.S. Wen, G. Gu, C.T. Lin, H. Claus, M.R. Norman, M. Randeria, J.C. Campuzano, Nature Phys. 6 (2010) 99.

[4] L. Balents, M.P.A. Fisher, C. Nayak, Int. J. Mod. Phys. B 12 (1998) 1033.

[5] P.W. Anderson, P.A. Lee, M. Randeria, T.M. Rice, N. Trivedi, F.C. Zhang, J. Phys. Condensed Matter 16 (2004) R755.

[6] Z. Tesanovic, Nature Phys. 4 (2008) 408.

[7] P.A. Lee, Science 321 (2008) 1306.

[8] L. Balents, Nature 464 (2010) 199.

[9] G. Kotliar, Phys. Rev. B 37 (1988) 3664.

[10] T. Senthil, M.P.A. Fisher, Phys. Rev. B 62 (2000) 7850.

[11] R. Moessner, S.L. Sondhi, Phys. Rev. Lett. 86 (2001) 1881.

[12] A.Y. Kitaev, Ann. Phys. (NY) 303 (2003) 2.

[13] O.I. Motrunich, T. Senthil, Phys. Rev. Lett. 89 (2002) 277004.

[14] M.A. Levin, X.G. Wen, Phys. Rev. B 71 (2005) 045110.

[15] X.G. Wen, Quantum Field Theory of Many-Body Systems: From the Origin of Sound to an Origin of Light and Electrons, Oxford University Press, New York, 2004.

[16] Z.Y. Meng, T.C. Lang, S. Wessel, F.F. Assaad, A. Muramatsu, Nature 464 (2010) 847.

[17] S. Yan, D.A. Huse, S.R. White, Science 332 (2011) 1173.

[18] H.C. Jiang, H. Yao, L. Balents, Phys. Rev. B 86 (2012) 024424.

[19] J.S. Helton, K. Matan, M.P. Shores, E.A. Nytko, B.M. Bartlett, Y. Yoshida, Y. Takano, A. Suslov, Y. Qiu, J.-H. Chung, D.G. Nocera, Y.S. Lee, Phys. Rev. Lett. 98 (2007) 107204.

[20] S. Yamashita, Y. Nakazawa, M. Oguni, Y. Oshima, H. Nojiri, Y. Shimizu, K. Miyagawa, K. Kanoda, Nature Phys. 4 (2008) 459.

[21] T.-H. Han, J.S. Helton, S. Chu, D.G. Nocera, J.A. Rodriguez-Rivera, C. Broholm, Y.S. Lee, Nature 492 (2012) 406.

[22] R. Shankar, Rev. Mod. Phys. 66 (1994) 129.

[23] P. Kopietz, L. Bartosch, F. Schütz, Introduction to the Functional Renormalization Group, Springer, Berlin, 2010.

[24] W. Metzner, M. Salmhofer, C. Honerkamp, V. Meden, K. Schönhammer, Rev. Mod. Phys. 84 (2012) 299.

[25] I. Dzyaloshinskii, Sov. Phys. JETP 66 (1987) 848.

[26] P. Lederer, G. Montambaux, D. Poilblanc, J. Phys. 48 (1987) 1613.

[27] J. Gonzalez, F. Guinea, M.A.H. Vozmediano, Phys. Rev. Lett. 79 (1997) 3514.

[28] N. Furukawa, T.M. Rice, J. Phys.: Condensed Matter 10 (1998) L381.

[29] N. Furukawa, T.M. Rice, M. Salmhofer, Phys. Rev. Lett. 81 (1998) 3195.

[30] B. Binz, D. Baeriswyl, B. Doucot, Eur. Phys. J. B 25 (2002) 69.

[31] A. Ferraz, Phys. Rev. B 68 (2003) 075115.

[32] D. Zanchi, H.J. Schulz, Phys. Rev. B 61 (2000) 13609.

[33] C.J. Halboth, W. Metzner, Phys. Rev. B 61 (2000) 7364. 
[34] C. Honerkamp, M. Salmhofer, N. Furukawa, T.M. Rice, Phys. Rev. B 63 (2001) 035109.

[35] H. Freire, E. Correa, A. Ferraz, Phys. Rev. B 78 (2008) 125114.

[36] A.A. Katanin, Phys. Rev. B 79 (2009) 235119.

[37] A.A. Katanin, A.P. Kampf, Phys. Rev. Lett. 93 (2004) 106406.

[38] D. Rohe, W. Metzner, Phys. Rev. B 71 (2005) 115116.

[39] Ar. Abanov, A.V. Chubukov, Phys. Rev. Lett. 84 (2000) 5608.

[40] Ar. Abanov, A.V. Chubukov, Phys. Rev. Lett. 93 (2004) 255702.

[41] M.A. Metlitski, S. Sachdev, Phys. Rev. B 82 (2010) 075128;

For a sign-problem-free quantum Monte-Carlo simulation of this low-energy theory, see also E. Berg, M.A. Metlitski, S. Sachdev, Science 338 (2012) 1606.

[42] K.B. Efetov, H. Meier, C. Pépin, Nature Phys. 9 (2013) 442, arXiv:1210.3276v2.

[43] M.A. Metlitski, S. Sachdev, Phys. Rev. B 82 (2010) 075127.

[44] D.F. Mross, J. McGreevy, H. Liu, T. Senthil, Phys. Rev. B 82 (2010) 045121.

[45] C. Drukier, L. Bartosch, A. Isidori, P. Kopietz, Phys. Rev. B 85 (2012) 245120.

[46] H. Freire, E. Correa, A. Ferraz, Phys. Rev. B 71 (2005) 165113.

[47] See, e.g M.E. Peskin, D.V. Schroeder, An Introduction to Quantum Field Theory, Perseus Books, Cambridge, 1995.

[48] V.S. de Carvalho, H. Freire, EPL 96 (2011) 17006.

[49] J. Sólyom, Adv. Phys. 28 (1979) 201.

[50] H. Freire, E. Correa, A. Ferraz, Phys. Rev. B 73 (2006) 073103.

[51] H. Freire, E. Corrêa, A. Ferraz, J. Phys. A: Math. Gen. 39 (2006) 7977.

[52] K.G. Wilson, M.E. Fisher, Phys. Rev. Lett. 28 (1972) 240.

[53] The systematic study of higher-order corrections in the $\epsilon$-expansion was performed in E. Brezin, J.C. Le Gillou, J. Zinn-Justin, Phys. Rev. D 9 (1974) 1121;

See also J. Zinn-Justin, Quantum Field Theory and Critical Phenomena, Oxford University Press, New York, 2002.

[54] H. Fukuyama, T.M. Rice, C. Varma, B.I. Halperin, Phys. Rev. B 10 (1974) 3775.

[55] J. Voit, Rep. Prog. Phys. 58 (1995) 977.

[56] L. Balents, M.P.A. Fisher, Phys. Rev. B 53 (1996) 12133.

[57] H.-H. Lin, L. Balents, M.P.A. Fisher, Phys. Rev. B 56 (1997) 6569. 